\title{
REVISÃO SISTEMÁTICA DA LITERATURA SOBRE A RELAÇÃO ENTRE INOVAÇÃO E DESEMPENHO FINANCEIRO DAS STARTUPS
}

\section{SYSTEMATIC LITERATURE REVIEW ON THE RELATIONSHIP BETWEEN INNOVATION AND FINANCIAL PERFORMANCE OF STARTUPS}

Versão do autor aceita publicada online: 27 jan. 2022

Publicado online: 16 fev. 2022

Como citar esse artigo - American Psychological Association (APA): Silva, L. S. C. V., Kaczam, F., Silva, D. J. C., Silva, B. N. \& Lucena, W. G. L. (2022). Revisão sistemática da literatura sobre a relação entre inovação e desempenho financeiro das startups. Exacta. DOI: https://doi.org/10.5585/exactaep.2022.20997.

\section{Luciana Santos Costa Vieira da Silva} luvcosta10@gmail.com https://orcid.org/0000-0002-9538-7150 http://lattes.cnpq.br/0903901167501516 Universidade Federal da Paraíba Mestra em Engenharia de Produção pela Universidade Federal de Santa Catarina, Doutora em Administração pela Universidade Federal de Santa Catarina e Pós-Doutoranda no Programa de Pós-Graduação em Contabilidade da Universidade Federal da Paraíba.

\section{Fabíola Kaczam}

fabiola.eng.prod.utfpr@gmail.com https://orcid.org/0000-0002-0460-9927

Universidade Federal de Santa Maria

Mestra em Engenharia de Produção pela Universidade Federal de Santa Maria e Doutoranda do Programa de Pós-Graduação em Administração da UFSM.

\section{Deoclécio Júnior Cardoso da Silva}

deocleciojunior2009@hotmail.com

https://orcid.org/0000-0002-2395-2878

Universidade Federal de Santa Maria

Mestre em Engenharia de Produção pela Universidade Federal de Santa Maria e Doutorando do Programa de Pós-Graduação em Administração da UFSM.

\section{Bruno Nogueira Silva}

b.nogueira.silva@gmail.com

https://orcid.org/0000-0002-9991-4595

Universidade Federal Rural do Semi-Árido

Mestrando do Programa de Pós-Graduação em Administração da UFERSA 


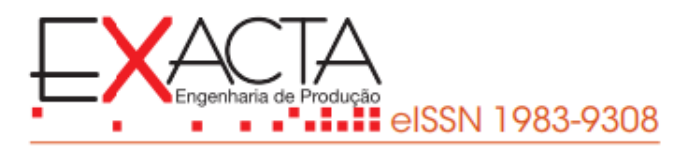

\section{Wenner Gláucio Lopes Lucena}

wdlucena@yahoo.com.br

https://orcid.org/0000-0002-2476-7383

Universidade Federal da Paraíba

Doutor em Ciências Contábeis pelo Programa Multiinstitucional e Inter-Regional de PósGraduação em Ciências Contábeis e Professor do Programa de Pós-graduação em Ciências Contábeis da UFPB.

\section{RESUMO}

O objetivo deste artigo é desenvolver uma Revisão Sistemática de Literatura (RSL) sobre a relação entre inovação e desempenho financeiro em startups. Como método de coleta foram selecionados 49 artigos nas bases de dados Scopus e Web of Science. Já o método de análise foi apoiado nas três leis da bibliometria clássica. O período de 2011 a 2020 foi definido para a elaboração deste estudo. Em termos teóricos e práticos há necessidade de as startups terem uma cultura organizacional que utilize práticas inovadoras e norteie o ambiente interno na adoção de comportamentos inovadores e com relação à sociedade, estreite as relações entre as universidades, incubadoras e fontes de financiamento com formação de alianças fortes para a promoção da inovação além de um amadurecimento maior nas redes de cooperação entre as empresas. A contribuição consiste em oferecer evidências sobre os resultados dos estudos, que podem sustentar a tomada de decisão de gestores de startups e gestores públicos, na criação de estratégias e políticas orientada a competitividade, como por exemplo, o estreitamento das relações entre empresas e universidades e a ampliação de redes de cooperação, conforme encontrado nas análises.

Palavras-Chave: Inovação. Startups. Desempenho. Revisão Sistemática de Literatura.

\section{ABSTRACT}

The aim of this article is to develop a Systematic Literature Review (RSL) on the relationship between innovation and financial performance in Startups. Forty-nine articles were selected from the Scopus and Web of Science databases. The analyzes were supported by the three laws of classical bibliometrics. The period from 2011 to 2020 was defined for the preparation of this study. There is a need for startups to adopt an organizational culture that adopts 


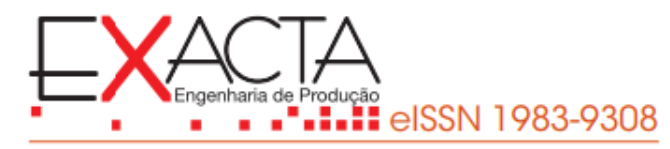

innovative practices and guides the internal environment in the adoption of innovative behaviors, as well as strengthening relationships between universities, incubators and funding sources with the formation of strong alliances to promote innovation in addition to greater maturation in cooperation networks between companies. The contribution consists of offering evidence on the results of the studies, which can support the decision-making of startup managers and public managers, in the creation of strategies and policies oriented towards competitiveness, such as, for example, the strengthening of relations between companies and universities and the expansion of cooperation networks, as found in the analysis.

Keywords: Innovation. Startups. Performance. Systematic Literature Review.

\section{INTRODUÇÃO}

A competitividade impulsionada, principalmente, pelo avanço tecnológico exige dos empreendimentos respostas flexíveis às mudanças do ambiente de negócios. Diante dessa realidade, as empresas buscam a inovação para obter vantagem competitiva (Zizlavsky, 2016). Inovar consiste em criar e implementar novos produtos, serviços e processos com foco na obtenção de melhorias significativas do desempenho organizacional (Choi \& Lee, 2016). A relação entre inovação e desempenho das empresas foi amplamente discutida em estudos anteriores (Clauss et al., 2019; Zhang et al., 2020). No entanto, os achados dos estudos dessa relação até o momento não se mostraram eficientes para determinados modelos de negócios, como por exemplo, as startups. Ries (2012) associa as startups ao empreendedorismo por oportunidade; empresas criadas para desenvolver novos produtos ou serviços sob condições de extrema incerteza e têm a inovação como o centro de suas operações.

No que diz respeito ao desempenho dessas empresas, de acordo com Godoi, Menolli \& Dionísio (2019), 18\% das startups encerram suas atividades em até dois anos, $67 \%$ em cinco anos e $74 \%$ após o quinto ano de operação. Contudo, outros estudos mostram que a inovação potencializa a lucratividade das empresas à medida em que facilita a produção e lançamento de novas marcas, o fortalecimento da posição de mercado, e o aumento da produtividade ou escalabilidade (Ali, 1994; Greve \& Taylor, 2000; Mai et al., 2019). 


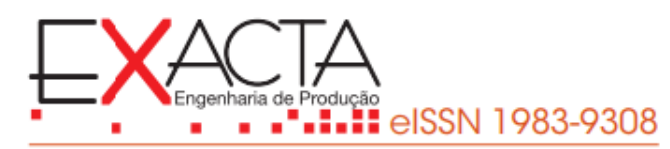

Sendo assim, ao considerar os fatores que compreendem a lucratividade, a qual pode vir a ser representada pelo desempenho financeiro superior, emerge a seguinte pergunta de pesquisa: "Qual a relação entre inovação e desempenho financeiro das startups?” Em resposta a esse questionamento o objetivo do presente artigo é desenvolver uma Revisão Sistemática de Literatura (RSL) sobre a relação entre inovação e desempenho financeirro em startups, a partir da produção científica contemplada pelas bases de dados Scopus e Web of Science.

A importância para se realizar a revisão sistemática pode ser justificada em função da obtenção de subsídios para fundamentar e implementar a prática baseada em evidências (DeLa-Torre-Ugarte-Guanilo, Takahashi \& Bertolozzi, 2011). Os resultados apresentados podem fundamentar a definição de categorias de análise para estudos qualitativos, ou na elaboração de hipóteses de estudos quantitativos.

O trabalho está estruturado em cinco seções: a primeira contemplou a introdução; na segunda seção está descrito referencial teórico que aborda os principais conceitos relacionados ao tema estudado; a terceira contém os procedimentos metodológicos utilizados, seguida pela quarta seção, na qual estão descritas a análise e discussão dos dados e a quinta corresponde às considerações finais da pesquisa.

\section{REFERENCIAL TEÓRICO}

Nessa etapa é apresentado o suporte teórico sobre o assunto abordado, de forma a dar sustentação às análises empíricas da Revisão Sistemática de Literatura (RSL), tecendo algumas considerações sobre os constructos inovação e desempenho, além de descrever brevemente sobre as startups.

\subsection{Inovação e Desempenho}

Falar sobre inovação nos dias de hoje é apresentar o que ocorre nos negócios e na vida de um modo geral em termos de novidade, pois a inovação é a expressão da mudança contínua e consistente. Assim, para entender o conceito de inovação, precisamos primeiro recorrer ao Manual de Oslo criado pela Organização para Cooperação e Desenvolvimento Econômico 


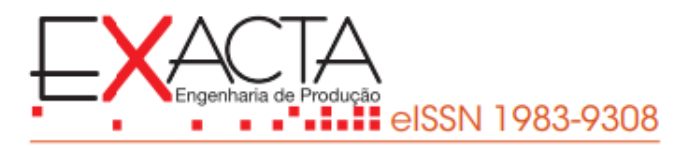

(OECD, 2005) que define inovação como: “a introdução de um bem ou serviço novo ou significativamente melhorado, no que se refere às suas características ou usos previstos, ou ainda, à implementação de métodos ou processos de produção, distribuição, marketing ou organizacionais novos ou significativamente melhorados". Nesse contexto, a inovação é a soma de conhecimentos, recursos e oportunidades ou necessidades em busca de um resultado satisfatório.

Para Winter (2006) a inovação pode percorrer uma trajetória natural a começar por micro mudanças, inovações menores, tentativas e erro, correção e falhas, solução de gargalos e ganhos de experiência e prática, começando a sanar os problemas críticos que estão evidentes. O caminho do progresso técnico é fruto das soluções dos problemas cotidianos. Mas é preciso considerar que é específico para cada organização e considerado um determinante chave do desempenho (Damanpour, 2010; Piening \& Salge, 2015). Assim, a inovação refere-se ao processo de implementação de novas ideias relacionadas ao desenvolvimento de tecnologias, processos e produtos.

De acordo com Gök \& Peker (2017) a inovação é um dos fenômenos interdisciplinares mais amplamente investigados e o impacto da inovação no desempenho da empresa tem sido de interesse de várias áreas por décadas. Nesse contexto, Bolivar-Ramos et al., (2012) afirmam que a inovação contribui para o aumento do desempenho empresarial, porém considerando o ambiente empresarial moderno, Tsai \& Yang (2014) e Zulu-Chisangaet Boso, Adeola \& Oghazi (2016) enfatizam o papel moderador da turbulência ambiental entre a inovação e o desempenho empresarial, explicando que produtos e serviços tornam-se rapidamente obsoletos em ambientes com mudanças rápidas.

Kluge, Meffert \& Stein (2000) sugeriram que a inovação está relacionada ao desempenho da empresa tanto no setor industrial quanto no setor de serviços e que a inovação tem uma influência muito grande no desempenho financeiro da empresa. Para Yildiz \& Karakas (2012), o desempenho da empresa é um conjunto de esforços feitos pela empresa com a finalidade de atingir os objetivos de negócios da mesma. Portanto, o desempenho geral da empresa depende da aplicação dos processos de negócios e da posição da empresa no mercado e, por isso, empresas que possuem uma boa posição no setor terão um desempenho que tende a aumentar. 


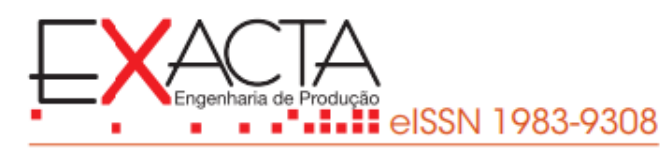

apoios instituídos para auxiliar as empresas nascentes nesse momento turbulento vivido não só no aspecto econômico, mas também no contexto social, demonstrando mais uma vez a importância da rede de colaboração para o sucesso do empreendedorismo e inovação.

Assim, diferentes estudos têm abordado a relação de startups com seu desempenho financeiro em casos como na identificação de padrões de financiamento de startups que influenciam no seu desempenho (Mittal \& Madan, 2020), o papel do investimento do capital de risco no crescimento e desempenho sustentável das startups (Jeong et al., 2020), o papel da orientação estratégica e do capital social dos fundadores no desempenho (Sohn, Hur \& Sohn, 2020), bem como o papel dos custos de design do modelo de negócios no estágio inicial das startups (Chammassian \& Sabatier, 2020).

Desse modo, alinhar as temáticas apresentadas se torna relevante, visto que esse modelo de empresa vem sendo um ator de desenvolvimento econômico, social e tecnológico de todo um ecossistema voltado para a inovação e o empreendedorismo.

\section{PROCEDIMENTOS METODOLÓGICOS}

A realização da Revisão Sistemática de Literatura (RSL) foi orientada pelo objetivo proposto: analisar a relação entre inovação e desempenho financeiro em startups, onde visa contribuir com a área de gestão alicerçado na seguinte pergunta de pesquisa: Qual a relação entre inovação e desempenho financeiro das startups? O estudo foi orientado em três etapas: i) estabelecimento da pergunta de pesquisa, ii) seleção e iii) classificação dos artigos científicos e disseminação do conhecimento.

Assim, a pergunta de pesquisa foi moldada de maneira ex-ante com suporte da literatura, a qual já determinava a existência de relação entre as variáveis inovação e desempenho (Steck \& Geenhuizen, 2016; Hojnik \& Ruzzier, 2016; Wilson \& Slobodzian, 2019; AlonsoMartínez, González-Álvarez \& Mariano Nieto, 2018; Yasar, Sezen \& Karakadilar, 2019; Hutahayan, 2020).

Com o objetivo de compor o corpus da pesquisa, foram realizadas buscas nas bases de periódicos Scopus e Web of Science. A escolha das bases de dados foi realizada em função de vincularem um grande volume de artigos científicos onde os estudos estejam relacionados 


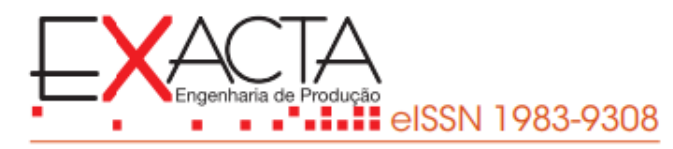

à pergunta de pesquisa. Para isso, foram usadas palavras-chave com uso de operadores booleanos tais como AND e OR.

A RSL foi conduzida com base nos protocolos de pesquisa de Tranfield, Denyer e Smart (2003), Kitchenham (2004) e Biolchini et al., (2005), sendo caracterizado como um estudo de natureza descritiva, de forma a identificar o estágio atual da produção científica sobre o relacionamento pesquisado e no rigor metodológico implícito no processo da RSL.

Nesse contexto, inicialmente foi construída uma string de busca alicerçada pelos critérios de inclusão e exclusão, pautando-se nos termos relacionados ao tema de pesquisa envolvendo as áreas de gestão. A string construída a partir das bases Scopus e Web of Science assim como a quantidade de resultados em termo do número de publicações em cada uma das bases levou em conta seis critérios de inclusão: (i) apenas artigos publicados em periódicos, (ii) artigos na língua inglesa, (iii) artigos publicados no período compreendido entre 2011 e 2020, (iv) artigos situados no primeiro quartil de citação dos Scimago Journal Ranking, (v) artigos cujos termos apareçam ao mesmo tempo na string de busca (aderência relacional), (vi) artigos aderentes à temática estudada após a sua leitura.

Os artigos foram localizados a partir de buscas em palavras-chave e termos que caracterizavam inovação, desempenho e firmas, tendo a busca sido realizada com os termos em inglês: innovation, performance e startups e suas variações. As strings usadas durante o processo de busca encontram-se evidenciadas na Tabela 1.

\section{Tabela 1}

\section{Strings de busca dos artigos do corpus}

\begin{tabular}{|c|c|c|}
\hline $\begin{array}{c}\text { Base de } \\
\text { Periódicos }\end{array}$ & String de busca & Resultados \\
\hline $\begin{array}{l}\text { Web of } \\
\text { Science }\end{array}$ & $\begin{array}{c}\text { TS }=(((\text { Innovation } *) \text { AND }(\text { Performance } *) \text { AND }(\text { Startup } * \text { OR } \\
\text { Incubator } * \text { OR Spin-off } * \text { OR Accelerator } *)))\end{array}$ & 391 \\
\hline Scopus & $\begin{array}{l}\text { TITLE-ABS-KEY(((Innovation) AND (Performance*) AND } \\
\text { (Startup* OR Incubator* OR Spin-off OR Accelerator* } *))\end{array}$ & 120 \\
\hline
\end{tabular}

Fonte: Os autores (2021).

A estratégia de busca foi definida em função de extrair artigos que efetivamente analisassem a relação proposta, sendo o título um critério de apoio e a composição de artigos da área de administração (business economic). Foram localizados 391 artigos na Web of Science e 120 
estudos no Scopus, totalizando 511 artigos. Após a revisão destes estudos, constatou-se que alguns não se adequavam à temática principal, à área de gestão.

Visando extrair os artigos diretamente vinculados a periódicos internacionais das áreas de inovação e desempenho em startups, e em decorrência do volume de artigos, foram criados novos filtros específicos para revistas vinculadas à inovação e gestão.

O corpus de pesquisa que compõe a RSL foi compilado em planilha eletrônica, destacando os elementos essenciais de cada artigo individualmente que contribuem para realização da metodologia de RSL. Foram extraídos dados dos artigos referentes a indicadores de citação e de conteúdo.

Em decorrência da abrangência desse tema e do volume de artigos encontrados nas bases de periódicos um conjunto de critérios de seleção que compreende o protocolo de pesquisa da RSL é apresentado por meio de um fluxograma disposto na Figura 1. 


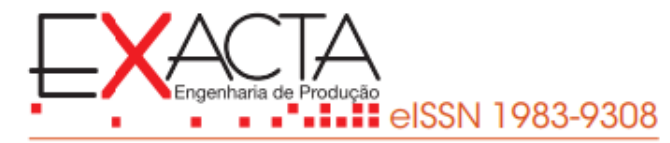

Figura 1

Sumarização do Protocolo da Revisão Sistemática de Literatura

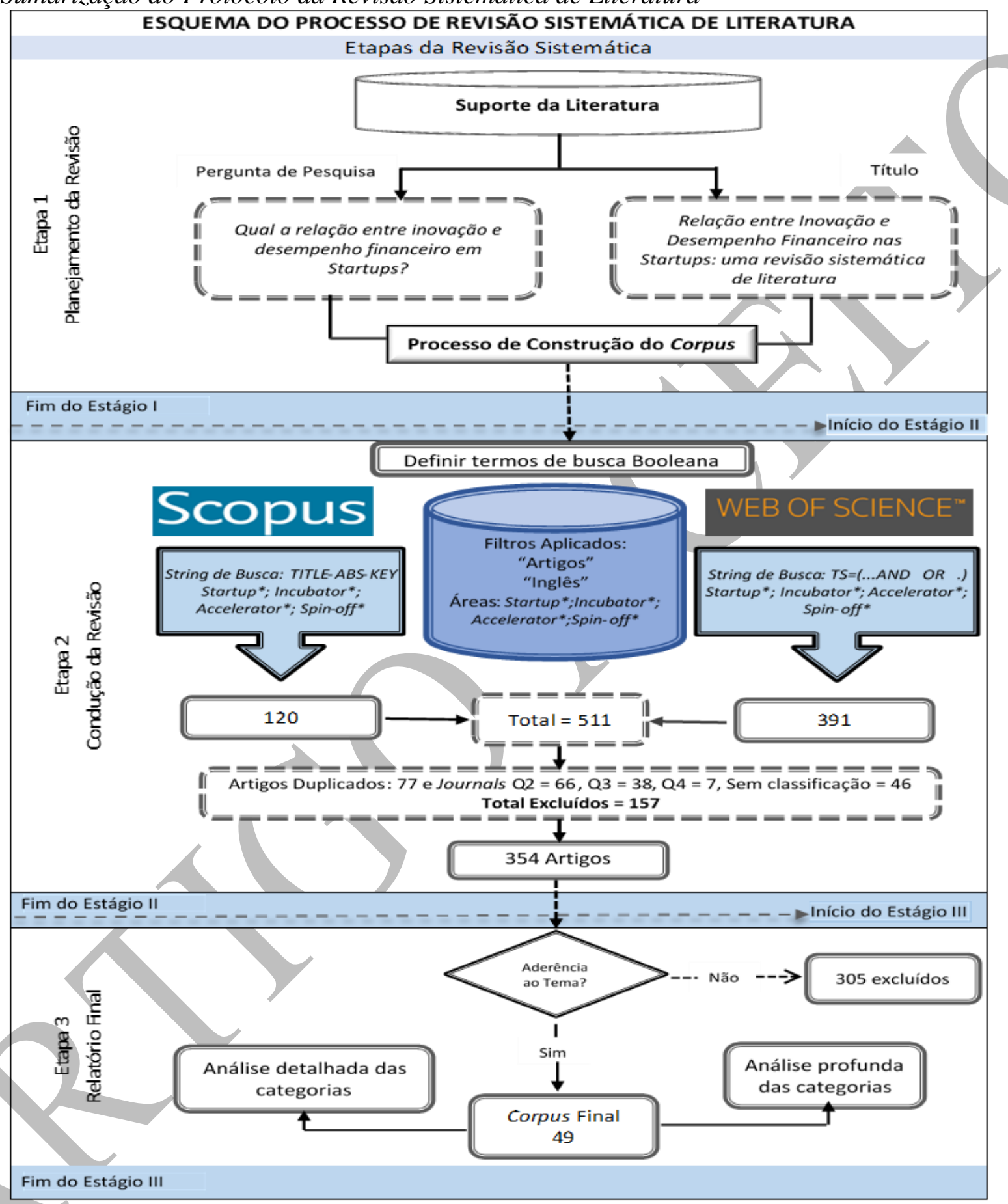

Fonte: Os autores (2021).

A construção do protocolo de pesquisa da RSL e seus resultados contribuem para avaliar os estudos selecionados. Vale salientar, que ao avaliar a aderência relacional à temática explorada, foram considerados apenas os artigos que exploravam de maneira conjunta os 
termos da string de busca, não sendo considerados os artigos que abordassem apenas um lado da string.

\section{APRESENTAÇÃO E ANÁLISE DOS DADOS}

Nesta seção, são apresentados os resultados encontrados a partir da análise do corpus onde busca-se avaliar a produtividade de autores e a frequência de ocorrência ou coocorrência de determinadas palavras em um texto.

\subsection{Distribuição Anual do Corpus}

Diante do corpus textual que compreende 49 artigos das bases de dados WoS e Scopus, a amostra abrange 29 periódicos e aproximadamente 140 autores e coautores, além de uma média de citação anual por documento publicado em torno de 3,17. Na Figura 2 pode-se visualizar as publicações dos artigos nos últimos 10 anos, considerando o período compreendido entre 2011 e 2020 em termos de quantidade e sua tendência.

\section{Figura 2}

Distribuição anual do corpus

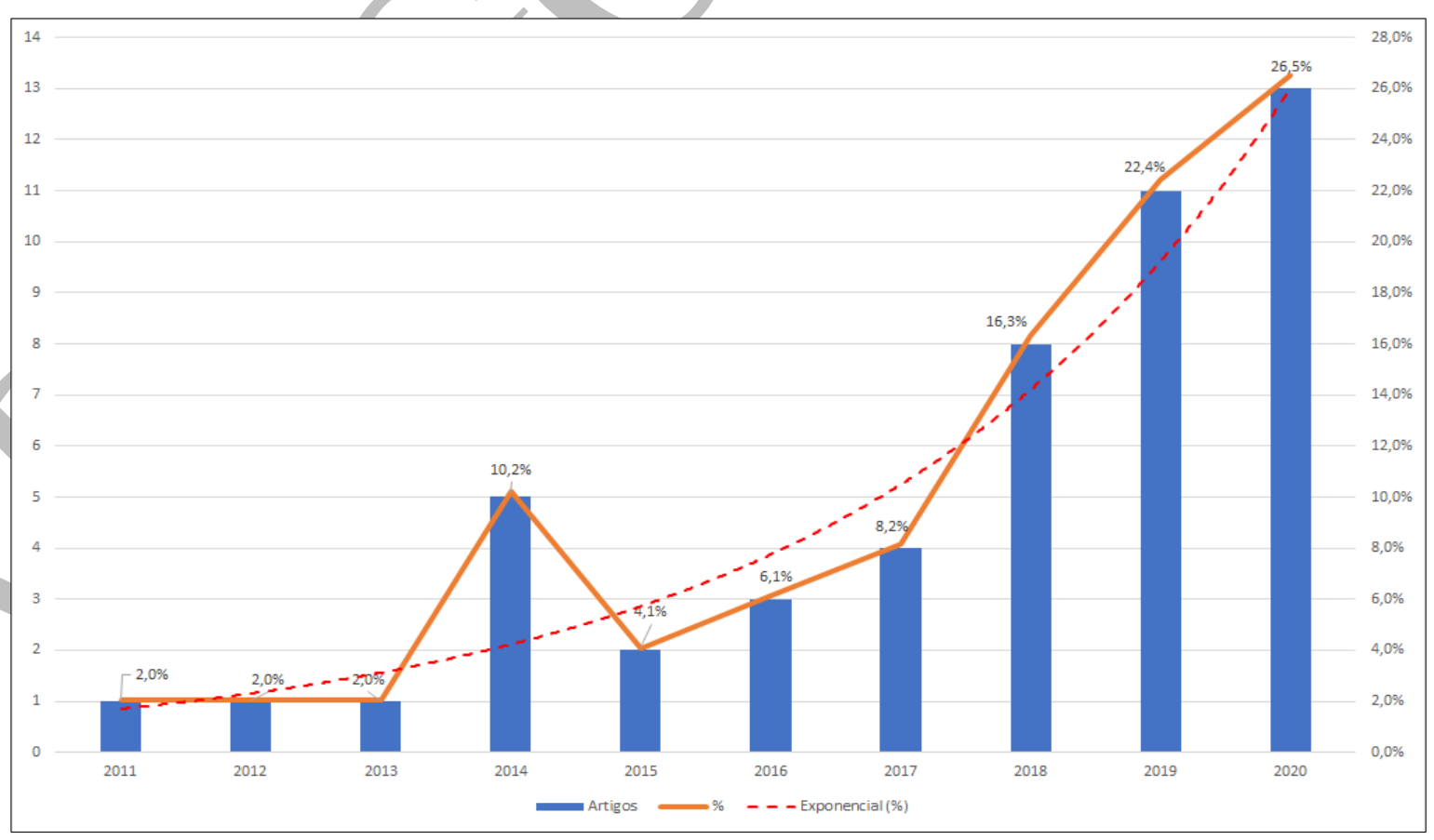

Fonte: Os autores (2021). 


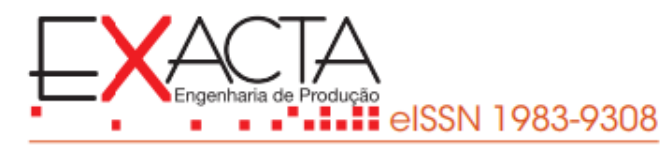

No período de 2011 a 2013 é possível observar que houve uma pequena quantidade de publicações de artigos que retratam a temática abordada, pois da amostra de 49 artigos, apenas 3 artigos foram publicados, representando um crescimento médio de $2 \%$ e uma média de citações igual a 5,08 nesse intervalo de tempo.

No período situado entre 2014 e 2017 percebe-se uma ocilação muito grande da quantidade de artigos publicados sobre essa tematica, uma vez que nesse mesmo período, publicou-se 14 trabalhos que correspondem a um crescimento médio de 7,14\% e uma média de citações igual a 6,05 nesse intervalo de tempo. Merece destaque o artigo descrito por Agarwal \& Shah (2014) intitulado como "Fontes de conhecimento de empreendedorismo: formação de empresas por acadêmicos, usuários e funcionários inovadores" com 81 citações e fora publicado no Research Policy. O objetivo desse trabalho foi identificar os fatores que afetam a capacidade de uma empresa de lucrar com a inovação. Os resultados evidenciaram o desenvolvimento de fatos estilizados e proposições preditivas relativas às diferenças nas contribuições inovadoras, papéis desempenhados na formação da dinâmica e evolução industrial e resultados de desempenho para startups decorrentes das três origens empresariais.

No período de 2018 a 2020 foram publicados 32 artigos centíficos sobre a referida temática, que corresponde a um crescimento médio igual a $21,8 \%$ de artigos publicados e uma média de citações de 5,53 nesse mesmo intervalo de tempo. Merece destaque em termos de quantidade de trabalhos publicados, o ano de 2020 que teve $26,5 \%$ do total de publicações nesse intervalo de tempo. O estudo que merece destaque nesse período é o de $\mathrm{Ng}$, AppelMeulenbroek, Cloodt \& Arentze (2019) intitulado: "Rumo a uma segmentação de parques científicos: um estudo de tipologia em parques científicos na Europa" com um total de 6 citações, sendo publìcado no periódico Research Policy. O objetivo desse trabalho foi apresentar uma pesquisa com 82 administradores de parques científicos na Europa buscando agrupá-los em três tipos clusters distintos: locais de pesquisa, cooperativas e incubadoras, buscando compreender e ao mesmo tempo avaliar o que eles oferecem aos pesquisadores, profíssionais e formuladores de políticas, como uma forma de comparar, comercializar e avaliar os parques científicos de forma mais adequada. 


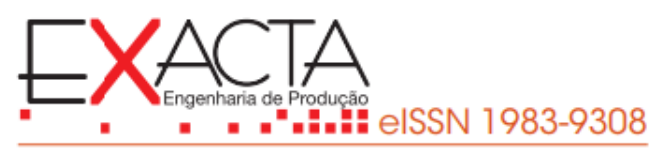

\subsection{Colaboração Científica entre Países}

Levando-se em conta que o avanço científico depende da interação entre os cientistas e que a colaboração entre duas ou mais pessoas seja caracterizada como um processo de interação social que pode acontecer de diversas formas e diferentes motivos, essa seção visa compreender e avaliar a maneira como os cientistas se comportam, se relacionam, se organizam e como transmitem informações entre si, com o objetivo de possibilitar que os estudos sejam realizados a partir da elevada diversidade de metodologias e pontos de vista intelectuais e culturais (Kretschmer, Liming \& Kundra, 2001).

Nesse contexto, optou-se pela utilização da coautoria, que apesar da opinião controversa de pesquisadores quanto ao seu uso como sinônimo de colaboração científica (Bordons \& Gómez, 2000; Lima, Velho \& Faria, 2007), dado que isso pode ser justificado, em função de apresentar vantagens ao dispor de dados que estão disponíveis publicamente, bem como permitir o exame de grandes amostras, o que possibilita resultados significativos ao comparar os estudos (Katz \& Martin, 1997; Meadows, 1999).

$\mathrm{Na}$ Figura 3 pode-se visualizar o comportamento das colaborações dos diferentes pesquisadores dos países avaliados, em função do número de documentos que foram publicados sobre a temática explorada. 


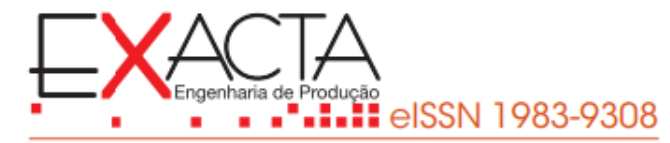

Figura 3

Top 20 em Colaboração Científica dos Autores e os Países Correspondentes (SCP e MCP)

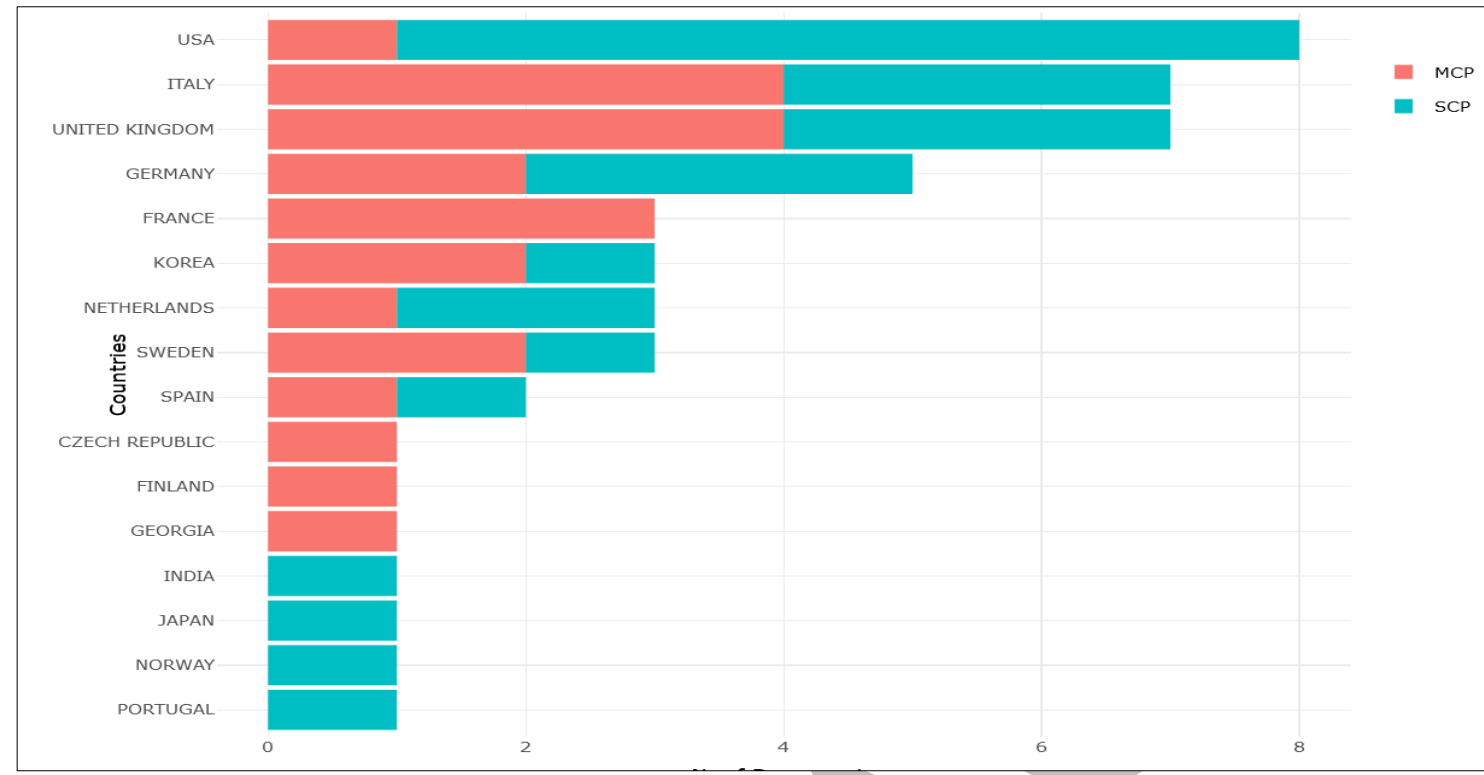

Fonte: Os autores (2021).

Por meio da Figura 3 é possível notar que as colaborações científicas foram divididas em dois tipos: aqueles artigos que foram publicados por autores de um único país (produção independente) - identificado pela sigla SCP (Single Country Publications), disposto na cor azul; e as produções com outros países (produção conjunta) - identificado pela sigla $M C P$ (Multiple Country Publications), disposto na cor vermelha.

Com relação à classificação adotada, entende-se como colaboração entre países, coautores de países distintos em relação ao autor principal, de maneira que cada artigo representa um país. Avaliando-se inicialmente somente os países que se destacaram realizando suas pesquisas sem qualquer tipo de cooperação científica com outros países (SCP) aponta-se: Índia, Japão, Portugal e Noruega, todos com (2,08\%). Por outro lado, os países que mais se evidenciaram realizando suas pesquisas de maneira cooperada com outros países (MCP) foram: Estados Unidos (16,67\%), Itália (14,58\%), Reino Unido (14,58\%), Alemanha $(10,42 \%)$ e França $(6,25 \%)$.

Em relação aos dois países de maior destaque que fazem colaboração em conjunto (Itália e

Reino Unido), pode-se destacar o trabalho desenvolvido por Colombo et al., (2015), da Universidade Politécnica de Milão - Itália, intitulado como "Hybrid alliances and radical 


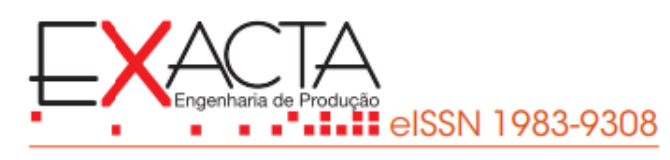

innovation: the performance implications of integrating exploration and exploitation", publicado no Journal of Technology Transfer. Outro trabalho de destaque foi desenvolvido por Wang et al., (2020) da Universidade de Coventry no Reino Unido, intitulado como "Capacities of Business Incubator and Regional Innovation Performance", que fora publicado no Technological Forecasting And Social Change, além do trabalho desenvolvido na Universidade de Kiel na Alemanha que fora desenvolvido por Walter et al., (2011), intitulado como "Championship Behaviors and Innovations Success: An Empirical Investigation of University Spin-Offs", e que fora publicado no periódico Journal of Product Innovation Management.

A justificativa para as parcerias denotadas anteriormente entre Itália, Alemanha e Reino Unido corroboram com o Global Innovation Index (2020), onde demonstra que tais países estão entre os 20 países que mais publicam artigos científicos e geraram patentes e ainda estão entre os 30 países de maior destaque de inovação, com destaque para o aumento de despesas com P\&D e na qualidade em inovação.

\subsection{Análise de Cocitação entre Autores}

A análise de cocitação realizada neste trabalho tem por objetivo mapear a frequência com que dois autores ou documentos da literatura pesquisada são citados de forma conjunta por algum outro autor mais recente (Small, 1973).

Assim, dois autores são cocitados caso exista um terceiro que os cita conjuntamente. Deste modo, Van Eck \& Waltman (2014) mostram que quanto maior for o número de documentos em que dois autores ou mesmo dois documentos sejam cocitados, mais forte também será a relação de cocitação éntre estes autores ou documentos. A análise de cocitação nesse artigo procura responder a seguinte pergunta secundária de pesquisa: “Quem são os autores mais cocitados?

Na Figura 4 evidencia-se de maneira pormenorizada a rede de cocitação entre os autores dos respectivos artigos, com base na quantidade de citações recebidas e a quantidade de laços relacionais entre os autores componentes do corpus.

Constata-se por meio da figura subsequente que, de forma geral, os estudos investigados com base na formulação dos três clusters apresentam uma relação teórica muito próxima, e que 
existe convergência nas citações de autores clássicos sobre a temática em estudo, evidenciadas pelo relacionamento entre eles.

\section{Figura 4}

Cocitação de autores do corpus

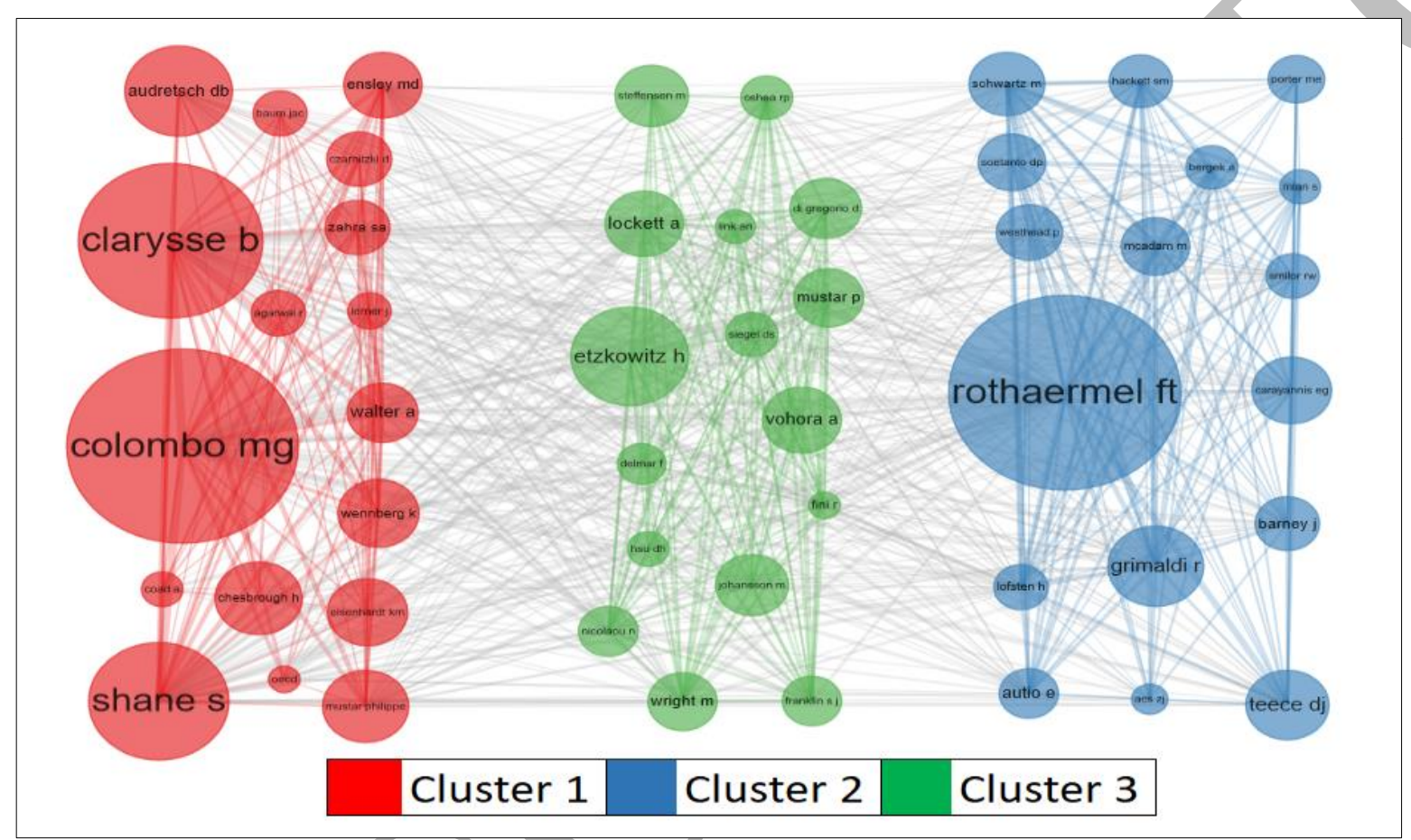

Fonte: Os autores (2021).

Considerou-se ainda a estatística de centralidade de intermediação que é uma medida onde consideram as formas como um pesquisador interage e se comunica com o restante dos demais pesquisadores da rede dos cinco principais autores de cada cluster formulado. A medida de centralidade considerada nessa pesquisa é a de centralidade global que é uma medida de centralidade de intermediação (betweeness centrality).

Ao analisar a quantidade de laços relacionais entre os autores mais cocitados de cada cluster formulado na Figura 4, percebeu-se que os autores de maior destaque do Cluster 1 pautandose na estatística betweeness centrality foi Colombo \& Piva (2012), com o trabalho intitulado como "Características genéticas das empresas e estratégias de ampliação de competências: uma comparação entre start-ups de alta tecnologia acadêmicas e não acadêmicas", onde obteve um valor igual a 63,551 para a estatística de betweeness centrality, um total de 634 


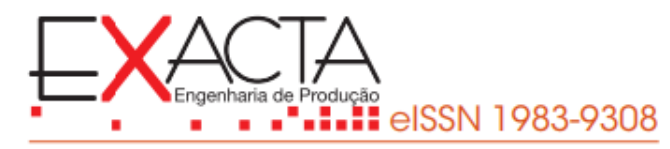

laços relacionais, além de um total de 33 cocitações por parte de outros autores. No Cluster 2 o destaque foi o trabalho desenvolvido por Rothaermel \& Thursby (2005), com um valor igual da estatística de betweeness centrality igual a 69,97, onde o trabalho intitulado como "Falência ou graduação da empresa incubadora?", obteve um total de 462 laços relacionais e foi cocitado 25 vezes por outros autores do corpus. No Cluster 3 o destaque foi o trabalho realizado por Etzkowitz (1998), intitulado como "As normas da ciência empreendedora: efeitos cognitivos das novas ligações universidade-indústria". O artigo obteve uma estatística de betweeness centrality igual a 13,631 e um valor igual a 363 laços relacionais, sendo 19 vezes cocitado pelos demais autores do corpus.

Vale salientar, que as cocitações analisadas anteriormente demostram a existência de similaridade, complementaridade, sobreposição ou mesmo contraposição de ideias em relação as referências cocitadas pelos autores destaques do corpus. Nesse sentido, os estudos analisados apresentam uma relação teórica muito próxima. Nesse sentido, existe uma convergência em termos de cocitação de autores clássicos sobre a temática em estudo, evidenciado assim um relacionamento entre eles. Além disso, os resultados dispostos na tabela anterior convergem com a proposta de estudo na relação entre inovação e desempenho em startups, pois as diversas publicações consultadas são específicas destas áreas.

Por fim, esse tipo de análise em termos dos autores do corpus permite a identificação das principais tendências teóricas desenvolvidas no campo já estudado, além de ajudar a desvendar os laços relacionais ou conexões entre os principais autores, tanto pela análise de conteúdo, quanto pela publicação conjunta dos autores.

\subsection{Nuvem e Rede de Palavras do Corpus}

A nuvem de palavras estimada nesse trabalho refere-se à organização e ao agrupamento gráfico das palavras em função da sua frequência ou ocorrência, o que possibilita de maneira rápida a identificação do conteúdo lexical bem como a centralidade representacional do corpus textual.

Observe por meio da Figura 5 a nuvem de palavras formulada a com base nos resumos dos artigos do corpus criadas a partir da análise lexical simples e que fora estimada usando o software Rstudio. Foram formuladas três nuvens de palavras que foram extraídas a partir dos 
objetivos dos trabalhos avaliados, das metodologias empregadas e dos principais resultados dos trabalhos, evidenciando assim os termos que mais saltam aos olhos do leitor.

\section{Figura 5}

Nuvens de Palavras do Corpus

\begin{tabular}{|c|c|c|}
\hline 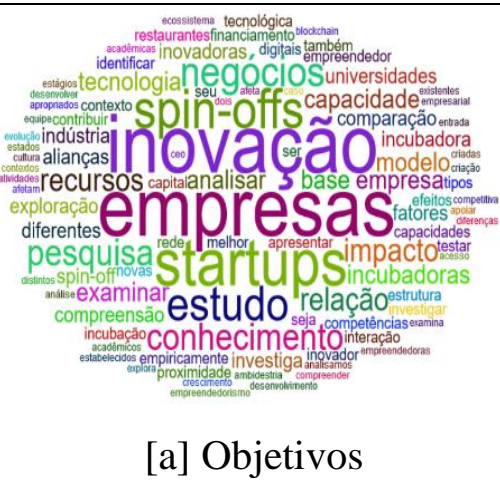 & [b] Métodos de Pesquisa & 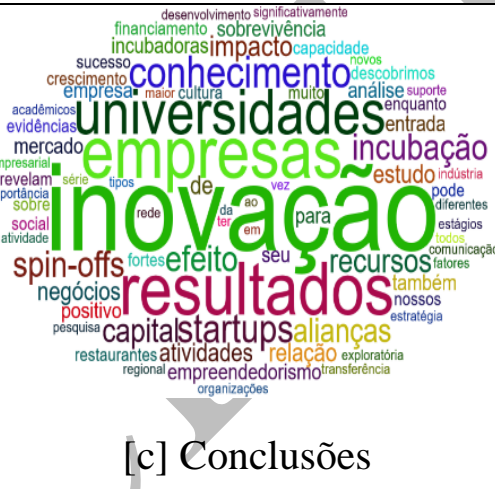 \\
\hline
\end{tabular}

Fonte: Os autores (2021).

Observe com base na nuvem de palavras disposta na Figura 5 que, em relação aos objetivos dos trabalhos do corpus, as palavras que mais aparecem são: "empresas", "inovação" e "startups" que foram o foco dos trabalhos. Vale ressaltar que o tamanho do nó estimado é proporcional à frequência de ocorrência das palavras. Assim, a relação dessas três palavras faz sentido com os propósitos dos trabalhos, pois as startups são empresas criadas com foco em inovação, ponto de partida dos autores para as pesquisas realizadas e publicadas nos artigos. No que diz respeito aos métodos de pesquisa adotados pelos artigos do corpus, percebe-se que o tipo de coleta dos dados com maior frequência de ocorrência foi a "survey", que está alinhado às pesquisas de natureza "quantitativa" e valendo-se de métricas de análises dos dados como "correlação", "análises de regressão", "análise fatorial" ou mesmo "modelagem de equações estruturais", pois a grande maioria dos trabalhos estão relacionados a estudos com países ou conglomerados de empresas.

Em relação a terceira nuvem de palavras relativo às conclusões dos trabalhos, percebe-se que as palavras que mais sobressaíram foram "inovação" que é um dos termos de busca pesquisados, "empresas" e "universidades", dado que retratam a relação entre universidadeempresa. É interessante observar que a maioria dos estudos sobre startups estão muito mais relacionados a incubadoras provenientes de Universidades do que de aceleradoras. 


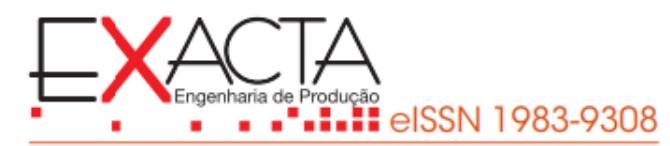

Uma outra forma de avaliar o comportamento das palavras refere-se aos seus relacionamentos a partir da rede de palavras. Nas visões de Van Eck \& Waltman (2014), a relação existente entre duas palavras-chave é determinada em função do número de artigos em uma mesma base de periódicos em que ambos ocorrem conjuntamente, em seu resumo ou mesmo na lista de palavras-chave.

A Figura 6 apresenta a relação entre as palavras-chave que permite avaliar os seus relacionamentos. Para tanto, utilizou-se o pacote bibliométrico "T-LabPlus versão 2021" com base nos resumos dos artigos, atribuindo-se o mesmo peso para cada palavra.

\section{Figura 6}

Rede de relacionamento entre as palavras do corpus

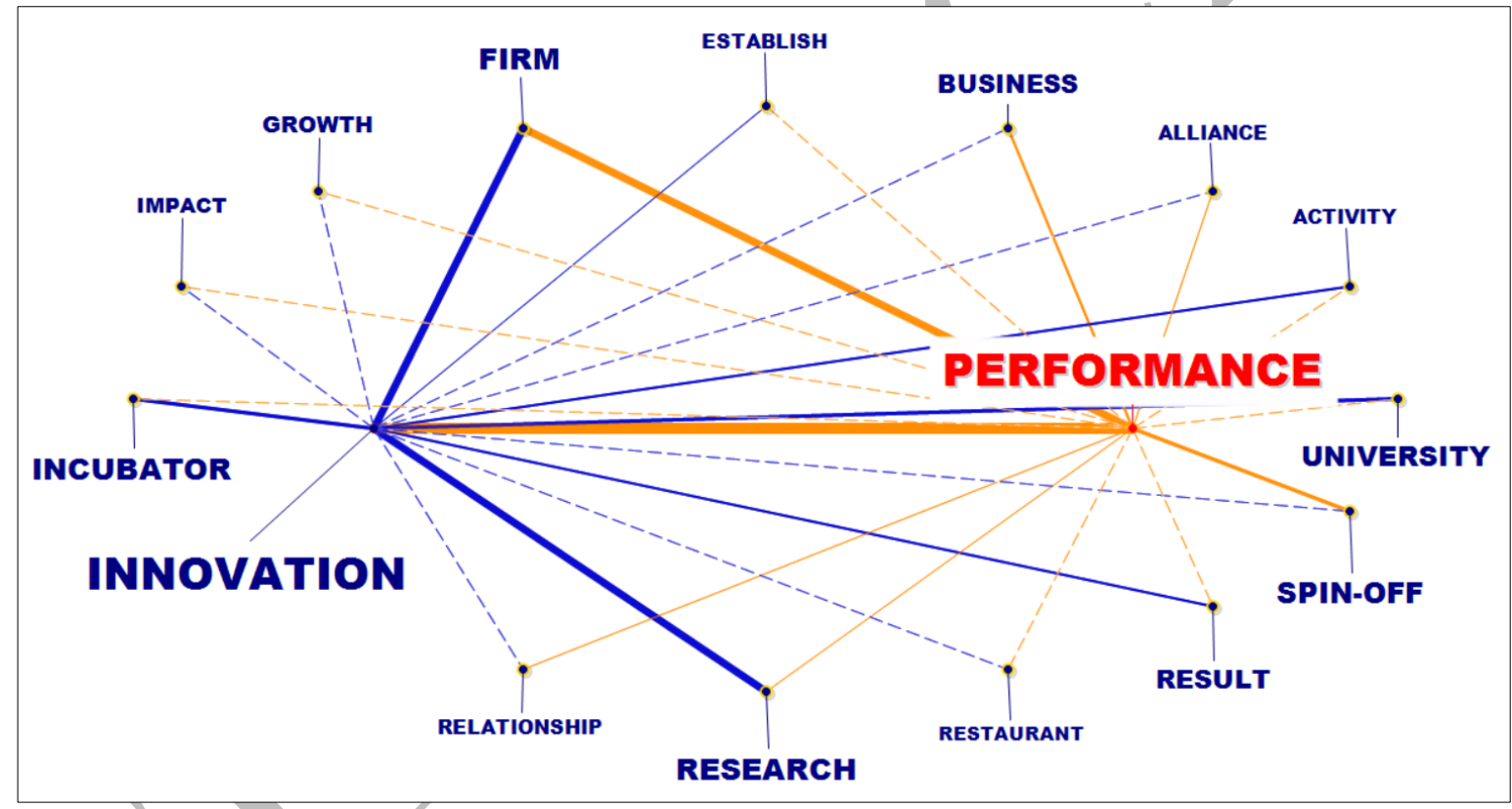

Fonte: Os autores (2021).

Conforme pode ser observado na Figura 6, há forte ligação entre a palavra performance e os termos inñovation, spin-off e establish. Esta relação é bem evidenciada pelo trabalho de Ferriani, Garnsey \& Lorenzoniy (2012), intitulado como "Continuity and change in a spinoff venture: the process of reimprinting" e publicado no Journal Industrial and Corporate Change. Tal artigo estudou a relação, normalmente de tensão, entre "herança organizacional e tecnológica" e busca por novidades; e apresentaram um modelo de aprendizagem intergeracional e desempenho de spin-off. Os autores reafirmaram ainda a importância das 


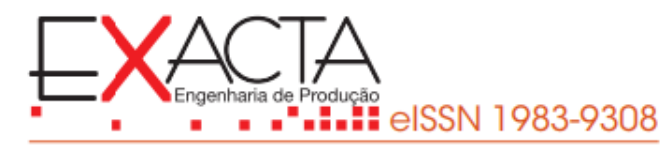

experiências passadas, e consequentemente das empresas mais experientes, no processo de continuidade e inovação no empreendimento de spin-off - sendo este considerado base para a inovação disruptiva.

É possível ainda vislumbrar significativa relação da palavra innovation com os vocábulos performance, business e restaurant, conforme exposto no estudo de Cho, Bonn \& Han (2020) - "Innovation ambidexterity: balancing exploitation and exploration for startup and established restaurants and impacts upon performance". O referido trabalho investigou se a ambidestria da inovação desempenha papéis diferentes na performance com relação aos estágios dos ciclos de vida de negócios de Startups e restaurantes estabelecidos. Seus achados corroboraram com a relação significativa e positiva entre a ambidestria da inovação e o desempenho de negócios dentro do contexto de restaurantes. Constatou-se assim, que restaurantes estabelecidos devem dedicar maior atenção à busca de inovação exploratória. Percebe-se ainda que ao avaliar a relação entre inovação e desempenho, encontra-se implícito o investimento em Pesquisa e Desenvolvimento (P\&D), dado que este aspecto pode ser justificado em função das empresas ao investem em P\&D necessitam de uma estratégia para a realização das mesmas, pois P\&D demanda aporte de recursos financeiros, tempo, pessoal que só faz sentido o investimento se a empresa tiver um propósito, uma meta, uma estratégia. Pode-se destacar ainda a relação existente no corpus entre desempenho e empreendedorismo entre os artigos do corpus textual, dado que a criação de startups passa pelo caráter empreendedor para a abertura de um negócio, bem como o propósito perseguido para o mesmo que é a obtenção de desempenho, de resultado, a partir do que se idealizou no negócio.

Por fim, as relações relatadas na figura anterior mostram que a criação e desenvolvimento de startups precisa de úm objetivo, uma estratégia empreendedora do gestor que precisa de conhecimento para se fazer pesquisa e desenvolvimento, a fim de se conseguir desempenho no processo de inovação.

\section{CONCLUSÕES E RECOMENDAÇÕES}

Este artigo teve como objetivo apresentar uma Revisão Sistemática de Literatura (RSL) sobre a relação entre inovação e desempenho em empresas privadas. Com a pretensão de contribuir 


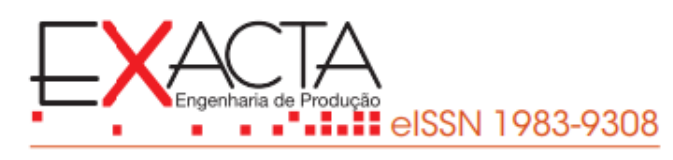

para o conhecimento científico da área de inovação e desempenho, este artigo foi construído tendo como alicerce uma questão de pesquisa definida ex-ante, elaborada a partir da literatura. Também foi criado um protocolo de pesquisa condizente com o rigor metodológico exigido na RSL (Tranfield, Denyer \& Smart, 2003), que resultou na composição do corpus de pesquisa.

Entre o período de 2011 a 2020 percebeu-se um aumento significativo de publicações sobre o tema. Isso demonstra o interesse em estudar essa relação entre inovação e desempenho financeiro das startups. Além disso, a pesquisa apresenta evidências que podem auxiliar gestores de startups na formulação de estratégias e políticas voltadas para a competitividade. As implicações apresentadas à comunidade acadêmica, gestores públicos e empresas privadas, orientam a necessidade de as startups adotarem uma cultura organizacional que adote práticas inovadoras e norteie o ambiente interno na adoção de comportamentos inovadores, bem como estreite as relações entre as universidades, incubadoras e fontes de financiamento (sejam elas públicas ou privadas), com formação de alianças fortes para a promoção da inovação. Os trabalhos que mais se destacaram apresentaram a necessidade de spin-offs e de um amadurecimento maior nas redes de cooperação entre as empresas.

Desse modo, com todas as análises explicitadas, pode-se entender que o objetivo da presente pesquisa foi alcançado. Este estudo contribui ao identificar o corpus da pesquisa sobre a relação entre inovação e desempenho, realizar um mapeamento e análise das características do campo, a partir de um problema de pesquisa específico. A contribuição consiste em oferecer evidências sobre os resultados dos estudos, que podem sustentar a tomada de decisão de gestores de startups e gestores públicos, na criação de estratégias e políticas orientada à competitividade, em conformidade com os achados nos estudos que compõem o corpus de pesquisa.

Como sugestões de pesquisas futuras pode-se indicar um estudo levando em consideração a relação abordada com o desempenho sustentável e social das startups, contribuindo com isso para a disseminação do conhecimento acerca deste tema emergente e relevante. Ainda, a verificação de estudos que abordem o desempenho não só financeiro, mas de inovação sustentável em diferentes atores que compõe um ecossistema de inovação. 


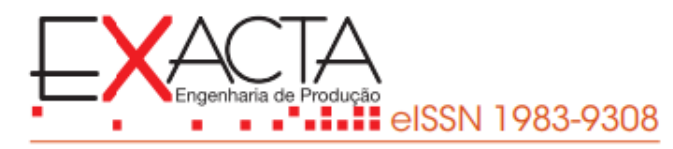

\section{REFERÊNCIAS}

ABSTARTUPS. Associação Brasileira de Startups. (2018).

https://abstartups.com.br/definicao-startups/>>. Acesso em: junho de 2021.

AGARWAL, R., \& SHAH, S. K. (2014). Knowledge sources of entrepreneurship: Firm formation by academic, user and employee innovators. Research Policy, 43(7), 1109-1133. https://doi.org/10.1016/j.respol.2014.04.012

ALI, A. (1994). Pioneering versus incremental innovation: Review and research propositions. Journal of Product Innovation Management, v. 11, n. 1, p. 46-61, https://doi.org/10.1111/1540-5885.1110046

ALONSO, D., GONZÁLEZ, N., \& NIETO, M. effect on entrepreneurship?", Journal of International Entrepreneurship (en prensa).

BEYHAN YASAR, N., SEZEN, B., \& KARAKADILAR, I. S. (2019). Mediating effect of continuous improvement on the relationship between innovation and financial performance.

Total Quality Management \& Business Excellence, 30(7-8), 893-907. https://doi.org/10.1080/14783363.2017.1353415

BIOlChinI, J., MiAN, P. G., NATALI, A. C. C., \& TRAVASSOS, G. H. (2005). Systematic review in software engineering. System Engineering and Computer Science Department COPPE/UFRJ, Technical Report ES, 679(05), 45.

BOLIVAR-RAMOS, M.T., GARCIA-MORALES, V.J. AND GARCIA-SANCHEZ, E. (2012). "Technological distinctive competencies and organizational learning: effects on organizational innovation to improve firm performance", Journal of Engineering and Technology Management, Vol. 29 No. 3, pp. 331-337. https://doi.org/10.1016/j.jengtecman.2012.03.006.

BORDONS, M., \& GÓMEZ, I. (2000). Collaboration networks in science. The web of knowledge: A festschrift in honor of Eugene Garfield, 197-213.

CACCIOLATTI, L., ROSLI, A., RUIZ-AlBA, J. L., \& CHANG, J. (2020). Strategic alliances and firm performance in startups with a social mission. Journal of Business Research, 106, 106-117. https://doi.org/10.1016/j.jbusres.2019.08.047 


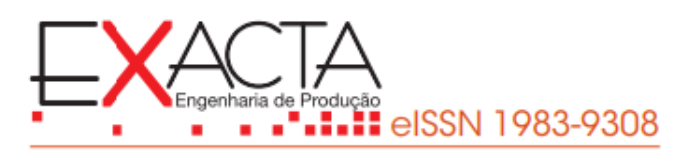

Journal of Innovation $\quad$ Management, 2050011. https://doi.org/10.1142/S1363919620500115

DE-LA-TORRE-UGARTE-GUANILO, M. C.; TAKAHASHI, R. F.; BERTOLOZZI, M. R. (2011). Revisão sistemática: noções gerais. Rev. esc. enferm. USP [online]. vol.45, n.5, pp.1260-1266. ISSN 0080-6234. DOI: https://doi.org/10.1590/S0080-62342011000500033. ETZKOWITZ, H. \& LEYDESDORFF, L. (1995). The triple helix-university-industrygovernment relations: a laboratory for knowledge-based economic development. EASST Review, 14(1): 14-19. SSRN: https://ssrn.com/abstract=2480085

ETZKOWITZ, H. \& LEYDESDORFF, L. (2000). The dynamics of innovation: from national systems and "mode 2 " to a triple helix of university-industry-government relations. Research Policy, 29:109-123. https://doi.org/10.1016/S0048-7333(99)00055-4 ETZKOWITZ, H. (1998). The norms of entrepreneurial science: cognitive effects of the new university-industry linkages. Research policy, 27(8), 823-833. https://doi.org/10.1016/S0048-7333(98)00093-6

FERRIANI, S.; GARNSEY, E. \& LORENZONI, G. (2012).Continuity and change in a spinoff venture: the process of reimprinting. Industrial And Corporate Change, [S.L.], v. 21, n. 4, p. 1011-1048, 8 mar. Oxford University Press (OUP). http://dx.doi.org/10.1093/icc/dts001.

GÖK, O., \& PEKER, S. (2017). Understanding the links among innovation performance, market performance and financial performance. Review of Managerial Science, 11(3), 605631. https://doi.org/10.1007/s11846-016-0198-8.

GREVE, H. R.; TAYLOR, A. Innovations as catalysts for organizational change: Shifts in organizational cognition and search. Administrative Science Quarterly, v. 45, n. 1, p. 54-80, 2000. DOI: https://doi.org/10.2307/2666979

HOJNIK, J. \& RUZZIER, M. (2016). The driving forces of process eco-innovation and its impact on performance: insights from Slovenia. Journal of Cleaner Production, 13:812-825, https://doi.org/10.1016/j.jclepro.2016.06.002.

HUTAHAYAN, B. (2020). The mediating role of human capital and management accounting information system in the relationship between innovation strategy and internal process performance and the impact on corporate financial performance. Benchmarking: An International Journal. https://doi.org/10.1108/BIJ-02-2018-0034. 


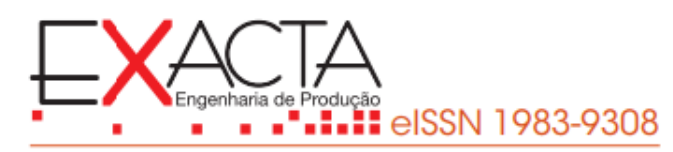

JEONG, J., KIM, J., SON, H., \& NAM, D. I. (2020). The role of venture capital investment in startups' sustainable growth and performance: Focusing on absorptive capacity and $\begin{array}{lllll}\text { venture } & \text { capitalists' } & \text { reputation. } & \text { Sustainability, } & 12(8),\end{array}$ https://doi.org/10.3390/su12083447.

JIMÉNEZ-JIMÉNEZ, D., \& SANZ-VALLE, R. (2011). Innovation, organizational learning, and performance. Journal of business research, 64(4), 408-417. https://doi.org/10.1016/j.jbusres.2010.09.010

KATZ, J. S., \& MARTIN, B. R. (1997). What is research collaboration?. Research policy, 26(1), 1-18. https://doi.org/10.1016/S0048-7333(96)00917-1.

KITCHENHAM, B. (2004). Procedures for performing systematic reviews. Keele, UK, Keele University, 33(2004), 1-26. ISSN: 1353-7776

KLUGE, J., MEFFERT, J., \& STEIN, L. (2000). The German road to innovation. The McKinsey Quarterly, 2(1), 99-105.

KOHLER, T. (2016). Corporate accelerators: Building bridges between corporations and startups. Business Horizons, 59(3), 347-357. https://doi.org/10.1016/jbushor.2016.01.008.

KORPER, A. K., PATRÍCIO, L., HOLMLID, S., \& WITELL, L. (2020). Service design as an innovation approach in technology startups: a longitudinal multiple case study. Creativity and Innovation Management, 29(2), 303-323. https://doi.org/10.1111/caim.12383

KRETSCHMER, H., LIMING, L., \& KUNDRA, R. (2001). Chinese-Indian-German collaboration results that provided the impetus for the foundation of COLLNET. Scientometrics, 52(3), 445-456. https://doi.org/10.1023/A:1014247900224.

KUCKERTZ, A., BRÄNDLE, L., GAUDIG, A., HINDERER, S., REYES, C. A. M., PROCHOTTA, A., ... \& BERGER, E. S. (2020). Startups in times of crisis-A rapid response to the COVID-19 pandemic. Journal of Business Venturing Insights, 13, e00169. https://doi.org/10.1016/j.jbvi.2020.e00169

LIMA, R. A. D., VELHO, L. M. L. S., \& FARIA, L. I. L. D. (2007). Indicadores bibliométricos de cooperação científica internacional em bioprospecção. Perspectivas em Ciência da Informação, 12, 50-64. https://doi.org/10.1590/s1413-99362007000100005.

MAI, A. N. et al. The lasting effects of innovation on firm profitability: panel evidence from a transitional economy, Economic Research-Ekonomska Istraživanja, v. 32, n. 1, p. 34173436, 2019 DOI: https://doi.org/10.1080/1331677X.2019.1660199. 


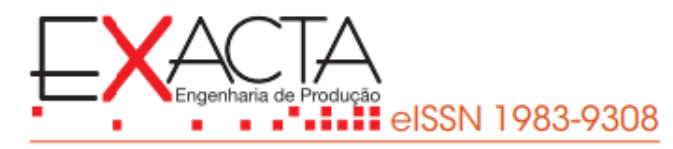

Management, 59, 39- 47. https://doi.org/10.1080/08956308.2016.1117329.

MANGIZA, P., \& BROWN, I. (2020, September). Requisite Skills Profile of Software Development Professionals for Startups. In Conference of the South African Institute of Computer Scientists and Information Technologists 2020 (pp. 102-109). https://doi.org/10.1145/3410886.3410904.

MEADOWS, C. (1999, May). Analysis of the Internet Key Exchange protocol using the NRL protocol analyzer. In Proceedings of the 1999 IEEE Symposium on Security and Privacy (Cat. No. 99CB36344) (pp. 216-231). IEEE. Https://doi.org/10.1109/SECPRI.1999.766916 MITTAL, T., \& MADAN, P. (2020). Impact of financing patterns on business performance of e-startups in India: a research model. International Journal of Business Innovation and Research, 21(4), 490-508. https://doi.org/10.1504/IJBIR.2020.105983

NG, W. K. B., APPEL-MEULENBROEK, R., ClOODT, M., \& ARENTZE, T. (2019). Towards a segmentation of science parks: A typology study on science parks in Europe. Research Policy, 48(3), 719-732. https://doi.org/10.1016/j.respol.2018.11.004

NiCOTRA, M., ROMANO, M., CASTROGIOVANNI, A., \& CORRENTE, S. (2020). Startups, Innovation and Performance: An Empirical Analysis in the Italian Context Using SMAA-S. In The Entrepreneurial Behaviour: Unveiling the cognitive and emotional aspect of entrepreneurship. Emerald Publishing Limited. https://doi.org/10.1108/978-1-78973-507920201008.

NORUZY A, DALFARD VM, AZHDARI B, NAZARI-SHIRKOUHI S, REZAZADEH A (2013) Relations between transformational leadership, organizational learning, knowledge management, organizational innovation, and organizational performance: an empirical investigation of manufacturing firms. Int J Adv Manuf Technol 64(5-8):1073-1085. https://doi.org/10.1007/s00170-012-4038-y.

OECD., Development (Paris)., Development. Development Centre, Organisation de coopération et de développement économiques (Paris), Statistical Office of the European COMMUNITIES, \& SOCIETY FOR INTERNATIONAL DEVELOPMENT. (2005). Oslo manual: Guidelines for collecting and interpreting innovation data (No. 4). Org. for Economic Cooperation \& Development. https://doi.org/10.1787/9789264213081-zh. 


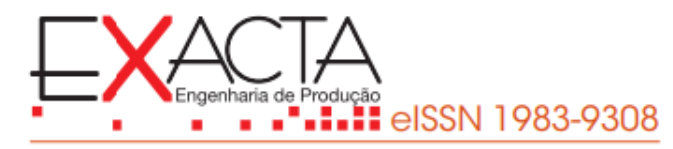

PARK, S. (2016). Differences in technology innovation R\&D performance creation behavior between for-profit institutions and not-for-profit institutions. Park Springer Plus, 5:451, p. 1-23, https://doi.org/10.1186/s40064-016-2092-x.

PIENING, E. P., \& SALGE, T. O. (2015). Understanding the antecedents, contingencies, and performance implications of process innovation: A dynamic capabilities perspective. Journal of Product Innovation Management, 32(1), 80-97. https://doi.org/10.1111/jpim.12225.

RIES, E. (2012). A Startup Enxuta. São Paulo: Leya, 275p.

ROTHAERMEL, F. T., \& THURSBY, M. (2005). Incubator firm failure or graduation?: The role of university linkages. Research policy, 34(7), 1076-1090. https://doi.org/10.1016/j.respol.2005.05.012

SMALL, H. (1973). Co-citation in the scientific literature: A new measure of the relationship between two documents. Journal of the American Society for information Science, 24(4), 265-269. https://doi.org/10.1002/asi.4630240406

STECK, P. E. \& GEENHUIZEN, M. S. VAN. (2016). The influence of international research interaction on national innovation performance: a bibliometric approach. Technological Forecasting \& Social Change, 110: 61-70, https://doi.org/10.1016/j.techfore.2015.09.017 TRANFIELD, D., DENYER, D. \& SMART, P. (2003). Towards a methodology for developing evidence-informed management knowledge by means of systematic review. British Journal of Management, 14: 207-222. https://doi.org/10.1111/1467-8551.00375.

TSAI, K. H. AND YANG, S. Y. (2014). "The contingent value of firm innovativeness for businessperformance under environmental turbulence", International Entrepreneurship and Management Journal, Vol. 10 No. 2, pp. 343-366. https://doi.org/10.1007/s11365-012-02254.

VAN ECK, N. J., \& WALTMAN, L. (2014). Visualizing bibliometric networks. In Measuring scholarly impact (pp. 285-320). Springer, Cham. https://doi.org/10.1007/978-3319-10377-8_13

WAlTER, A., PARBOTEEAH, K. P., RIESENHUBER, F., \& HOEGL, M. (2011). Championship behaviors and innovations success: An empirical investigation of university spin-offs. Journal of product innovation management, 28(4), 586-598. https://doi.org/10.1111/j.1540-5885.2011.00826.x 
WANG, Z., HE, Q., XIA, S., SARPONG, D., XIONG, A., \& MAAS, G. (2020). Capacities of business incubator and regional innovation performance. Technological Forecasting and Social Change, 158, 120125. https://doi.org/10.1016/j.techfore.2020.120125

WILSON, G. A., \& SLOBODZIAN, A. D. (2019). Enhancing financial performance by pharmacy innovation. Canadian Journal of Administrative Sciences/Revue Canadienne des Sciences de l'Administration, 37(4), 459-467. https://doi.org/10.1002/cjas.1562

WINTER, S. G. (2006). Toward a neo-Schumpeterian theory of the firm. Industrial and Corporate Change, 15(1), 125-141. https://doi.org/10.1093/icc/dtj006.

YILDIZ, S., \& KARAKAS, A. (2012). Defining methods and criteria for measuring business performance: A comparative research between the literature in Turkey and foreign. Procedia - Social and Behavioral Sciences, 58, 1091-1102. https://doi.org/10.1016/j.sbspro.2012.09.1090.

ZHANG, Y. et al. (2020). Effects of firm, industry, and country-level innovation on firm performance. Marketing Letters, v. 31, p. 231-245, 2020. DOI: https://doi.org/10.1007/s11002-020-09530-y

ZIZLAVSKY, O. (2016). Innovation performance measurement: research into Czech business practice. Economic research-Ekonomska istraživanja, 29(1), 816-838. DOI: https://doi.org/10.1080/1331677X.2016.1235983

ZULU-CHISANGA, S., BOSO, N., ADEOLA, O., \& OGHAZI, P. (2016). Investigating the path from firm innovativeness to financial performance: The roles of new product success, market responsiveness, and environment turbulence. Journal of Small Business Strategy, 26(1), 51-68. 